\title{
Coalitional Games in Receiver Cooperation for Spectrum Sharing
}

\author{
Suhas Mathur, Lalitha Sankaranarayanan and Narayan B. Mandayam \\ WINLAB Dept. of Electrical and Computer Engineering \\ Rutgers University, Piscataway, NJ \\ \{suhas, lalitha, narayan\}@winlab.rutgers.edu
}

\begin{abstract}
The issue of sharing spectrum through receiver cooperation in wireless networks is studied under the framework of coalitional game theory. In particular, we consider two illustrative network models: (1) a Gaussian interference channel with receiver cooperation and (2) a multiple access channel (MAC) with linear multiuser detection. Allowing any arbitrary sharing of the sum-rate achieved by a coalition between member links in a Gaussian interference channel, it is shown that the grand coalition (coalition of all receivers) maximizes spectrum utilization and is also stable. For the linear MMSE multiuser detector, it is shown that the grand coalition is always stable and sum-rate maximizing, while for the decorrelating multiuser detector, the above observation is shown to be true only in the high SNR regime. Finally, transmitter cooperation in the context of a Gaussian interference channel is discussed, with focus on some open problems and a simplified framework for the resulting coalitional game is proposed.
\end{abstract}

\section{INTRODUCTION}

The sharing of spectrum has been at the forefront of radio regulation and efficient system design since the earliest days of wireless. Inefficient spectrum sharing implies poor use of the wireless medium resulting in higher infrastructure costs, fewer supported users and lower communication rates. A number of approaches have been proposed for dealing with the spectrum sharing problem. These range from classical resource allocation techniques that do power control [1], channel allocation [2] and scheduling [3], all the way to transmitter [4], [5] and receiver cooperation [6]. The range of network models that represent such a scenario include the interference channel as well as the multiple access channel (MAC). Given that devices sharing the spectrum may differ vastly in their modulation schemes and protocols, especially in the unlicensed spectrum bands, receiver cooperation may only be feasible if one could find ways to jointly process their signals. A spectrum server (such as the one proposed in [7] and [8]) can serve as a central entity that enables disparate devices to jointly decode their signals. Specifically, the receivers of independent links in a shared spectrum environment can relay their received signals to the spectrum server which can then jointly decode them.

In this context, we are interested in determining the type of cooperative coalitions that devices should form with the receivers of other devices sharing the spectrum. One could also pose this question in the context of transmitter cooperation.
In this paper, we analyze the effect of cooperation under the framework of coalitional game theory to address the above questions through the following illustrative network models:

- A Gaussian interference channel (IC) with receiver cooperation [9]

- A multiple access channel (MAC) with linear multiuser detection.

We focus on the class of coalitional games in characteristic function form [10]. These games allow a coalition of cooperating users to be characterized by a single number, the value of the coalition, which does not depend upon the actions of users outside the coalition. For example, the value of a coalition could be the sum-rate achieved by jointly decoding all users in that coalition. Further, when users in a coalition are allowed an arbitrary apportioning of its value, the game is said to have transferable payoff. We find that receiver cooperation in an interference channel falls into this class of games while linear multiuser detection in a MAC results in games with non-transferable payoff. A natural question that arises in transferable payoff games is how these users should apportion the available sum-rate among themselves.

Cooperating receivers in an interference channel that decide to jointly decode their received signals may be treated as the distributed antennas of a single receiver. This treatment permits the interference channel to be viewed as a singleinput, multiple-output multiple access channel (SIMO-MAC). In section III, we consider such a model, where receiver cooperation converts an interference channel for cooperating links into a SIMO-MAC channel. In this context, we study what are the stable coalitions that can form. Here, by stability we mean no subset of links has incentive to break away from the coalition structure. A related question is whether a coalition that maximizes the sum rate can be stable. We also address the issue of allocation of rates to the members of these stable coalitions by proposing apportioning strategies as solutions to a bargaining game. In section IV, we investigate the stable coalitions that are likely to result when linear multiuser detectors are employed in a MAC. In each case, we present our results and illustrate with examples. Finally we discuss transmitter cooperation in the context of the model in section III and raise some issues and open problems. 


\section{Coalitional Games}

In this section we briefly review aspects of coalitional game theory [11] in the context of our problem. We consider coalitional games in which every coalition is ascribed a single number, interpreted as the payoff available to the coalition. The share of the payoff received by players in a coalition is called a payoff vector. When there are no restrictions on how this payoff may be apportioned between members, the game is said to have transferable payoff.

Definition 1: A coalitional game with transferable payoff $\langle\mathcal{S}, v\rangle$ consists of

- a finite set $\mathcal{S}$ of $M$ players,

- a function $v$ that associates with every non-empty subset $\mathcal{G}$ (a coalition) of $\mathcal{S}$, a real number $v(\mathcal{G})$ (the value of $\mathcal{G}$ ) with $v(\{\phi\})=0$

In general, the value of a coalition may not lend itself to arbitrary apportioning and the nature of the game may place restrictions on the ways that value can be apportioned. Such a game is said to have non-transferable payoff. As we shall see in the following sections, receiver cooperation with ideal joint decoding can be modeled as a coalitional game with transferable payoff while linear multiuser detection in the context of a MAC must be treated as a non-transferable payoff game. Further, receiver cooperation, whether through ideal joint decoding or through multiuser detection, results in coalitions where the value of each coalition is not influenced by the actions of players outside that coalition. This is because the maximum sum-rate $v(\mathcal{G})$ achievable by such a coalition $\mathcal{G}$ depends on the players in $\mathcal{G}^{c}$ only through the interference offered by them, which in turn is independent of coalitions formed within $\mathcal{G}^{c}$. In general, however, $v(\mathcal{G})$ may depend upon the actions of links outside the coalition $\mathcal{G}$. As we shall see in section 5, transmitter cooperation in an interference channel leads to such a situation.

Definition 2: A coalitional game with transferable payoff is said to be superadditive if for any two disjoint coalitions $\mathcal{G}_{1}, \mathcal{G}_{2} \subseteq S, v\left(\mathcal{G}_{1} \cup \mathcal{G}_{2}\right) \geq v\left(\mathcal{G}_{1}\right)+v\left(\mathcal{G}_{2}\right)$

Definition 3: A coalitional game with transferable payoff is said to be cohesive if the value of the coalition formed by the set of all players $\mathcal{S}$ (the grand coalition) is at least as large as the sum of the values of any partition of $\mathcal{S}$, i.e.

$$
v(\mathcal{S}) \geq \sum_{k=1}^{K} v\left(\mathcal{S}_{k}\right)
$$

for every partition $\left\{\mathcal{S}_{1}, \ldots, \mathcal{S}_{K}\right\}$ of $\mathcal{S}$.

A superadditive coalitional game is cohesive. In this sense, superadditivity is a stronger condition than cohesiveness but is often easier to observe. A transferable payoff game that is cohesive has the grand coalition as the optimal coalition structure (partition of links into coalitions) [11]. We remark that, without cohesiveness, finding the optimal coalition structure is an $\mathcal{N} \mathcal{P}$-complete problem [12]. This is because the number of possible coalition structures, given by the Bell number, grows exponentially fast with the number of players $M$.

Definition 4: For any coalition $\mathcal{G}$, a vector $\underline{x}_{\mathcal{G}}=\left(x_{m}\right)_{m \in \mathcal{G}}$ of real numbers is a $\mathcal{G}$-feasible payoff vector if $x(\mathcal{G})=$ $\sum_{m \in \mathcal{G}} x_{m}=v(\mathcal{G})$. The $\mathcal{S}$-feasible payoff vector is referred to as a feasible payoff profile.

Of all possible coalition structures that can form, the coalitions that are stable, that is, those in which no set of players (either from within or from across coalitions) have incentives to leave, are of most interest. The set of such stable coalitions comprises a core, defined formally below.

Definition 5: The core, $C(v)$, of a coalitional game with transferable payoff, $\langle\mathcal{S}, v\rangle$, is the set of feasible payoff profiles $\underline{x}_{\mathcal{S}}$ for which there is no coalition $\mathcal{G} \subset \mathcal{S}$ and a corresponding $\mathcal{G}$-feasible payoff vector $\underline{y}_{\mathcal{G}}=\left(y_{m}\right)_{m \in \mathcal{G}}$ such that $y_{m}>x_{m}$ for all $m \in \mathcal{G}$.

From definition 5, it follows that the set of feasible payoff profiles in the core satisfy

$$
v(\mathcal{G}) \leq x(\mathcal{G}) \text { for every coalition } \mathcal{G} \subset \mathcal{S}
$$

We note that this condition is equivalent to definition 5 . This is because in a game with transferable payoff if there exists a coalition $\mathcal{G}$ with $v(\mathcal{G})>x(\mathcal{G})$ then we can always find a $\mathcal{G}$-feasible payoff vector $\underline{y}_{\mathcal{G}}$ such that $y_{m}>x_{m}$, for all $m \in \mathcal{G}$. Such an assignment can result, for instance, when the $\mathcal{G}$-feasible payoff vector $y_{\mathcal{G}}$ is constructed by assigning to each link $m \in \mathcal{G}$, the payoff $\bar{x}_{m}$ and then uniformly apportioning the surplus payoff $v(\mathcal{G})-x(\mathcal{G})$ between links in $\mathcal{G}$.

\section{RECEIVER COOPERATION GAMES IN GAUSSIAN INTERFERENCE CHANNELS}

We consider an interference channel of $M$ communication links, each formed by a single transmitter-receiver pair, coexisting in the same shared spectrum [13]. We assume that every transmitter has a single antenna. $\mathcal{X}_{i}$ is the input alphabet of the transmitter of link $i$ and $\mathcal{Y}_{i}$ is the output alphabet at the corresponding receiver. We denote by $\mathcal{S}=\{1,2, \ldots, M\}$ the set of all links and write $X_{\mathcal{G}}=\left\{X_{i}: i \in \mathcal{G}\right\}$ for all $\mathcal{G} \subseteq \mathcal{S}$ and $\mathcal{G}^{c}$ as the complement of $\mathcal{G}$ in $\mathcal{S}$. We consider an additive white Gaussian noise channel with flat fading. The received signal at the receiver of link $i$ is given by

$$
Y_{i}=\sum_{k=1}^{M} h_{k, i} X_{k}+Z_{i}
$$

where $X_{i} \in \mathcal{X}_{i}, Y_{i} \in \mathcal{Y}_{i}$, and $h_{k i}$ is the channel gain between the transmitter of link $k$ to the receiver of link $i$ and is assumed known at the receiver. The noise entries $Z_{i} \sim \mathcal{C N}(0,1)$, are independent, circularly symmetric, complex Gaussian random variables with zero mean and unit variance, for all $i$. The power constraint at the $i^{\text {th }}$ transmitter is

$$
E\left|X_{i}\right|^{2} \leq P_{i} \quad i \in \mathcal{S}
$$


The capacity region of the interference channel that results when links do not cooperate is, in general, unknown. For the interference channel considered here, we assume that the transmitters employ Gaussian signaling subject to a power constraint (4). We also assume that receivers that choose to cooperate communicate with one another via a central spectrum server. Further, we assume that the transmitters do not cooperate. This models a variety of practical networks operating in the unlicensed bands where the receivers can communicate via a backbone network while the wireless transmitters, in general, cannot. For the input signaling considered, a coalition of cooperating receivers treats signals from transmitters outside the coalition as additive white Gaussian noise. Such a coalition can be modeled as a single-input, multiple-output multiple access channel (SIMO-MAC), the capacity region of which is known [14] and is achieved by the Gaussian input signaling chosen.

We define the value $v(\mathcal{G})$ of a coalition of receivers $\mathcal{G}$ as the maximum sum-rate achievable by the links corresponding to receivers in $\mathcal{G}$. For the channel model considered, $v(\mathcal{G})$ is then the mutual information between the transmitters and receivers in $\mathcal{G}$ given as

$$
v(\mathcal{G})=\max _{\underline{R}_{\mathcal{G}} \in \mathcal{C}_{\mathcal{G}}} \sum_{i \in \mathcal{G}} R_{i}=\max _{p_{X_{\mathcal{G}}}} I\left(X_{\mathcal{G}} ; Y_{\mathcal{G}}\right),
$$

where $\underline{R}_{\mathcal{G}}=\left(R_{i}\right)_{i \in \mathcal{G}}$ is the vector of rates for links in $\mathcal{G}$ and $\mathcal{C}_{\mathcal{G}}$ is the capacity region of the SIMO-MAC formed by the users in $\mathcal{G}$. The value $v(\mathcal{G})$ of a coalition $\mathcal{G}$ can be apportioned between its members in any arbitrary manner. Depending on its allocated share of $v(\mathcal{G})$, a receiver may decide to break away from the coalition $\mathcal{G}$ and join another coalition where it achieves a greater rate. We model the problem of determining the stable coalitions and the resulting rate allocations for the interference channel as a coalitional game with transferable payoff and refer to this game as the receiver cooperation interference channel game [9]. Using the results from section II we state the following two theorems (the proofs are given [9])

Theorem 6: The grand coalition maximizes spectrum utilization in the receiver cooperation interference channel game.

Theorem 7: The receiver cooperation interference channel game with transferable utility has a non-empty core.

Further, we have shown in [9] that every point on the dominant face (sum-rate maximizing face of the capacity polytope $\mathcal{C}_{\mathcal{S}}$, corresponding to the capacity region that results when all links cooperate to form a grand coalition) of the SIMO-MAC capacity region $\mathcal{C}_{\mathcal{S}}$ corresponds to a feasible rate payoff profile that lies in the core. Thus, the core for the interference channel coalitional game is not only non-empty but is, in general, also non-unique.

\section{Fair Allocations}

The core of the receiver cooperation interference channel game being non-unique, a natural question that arises is what allocation of rates must be chosen from all the payoff profiles
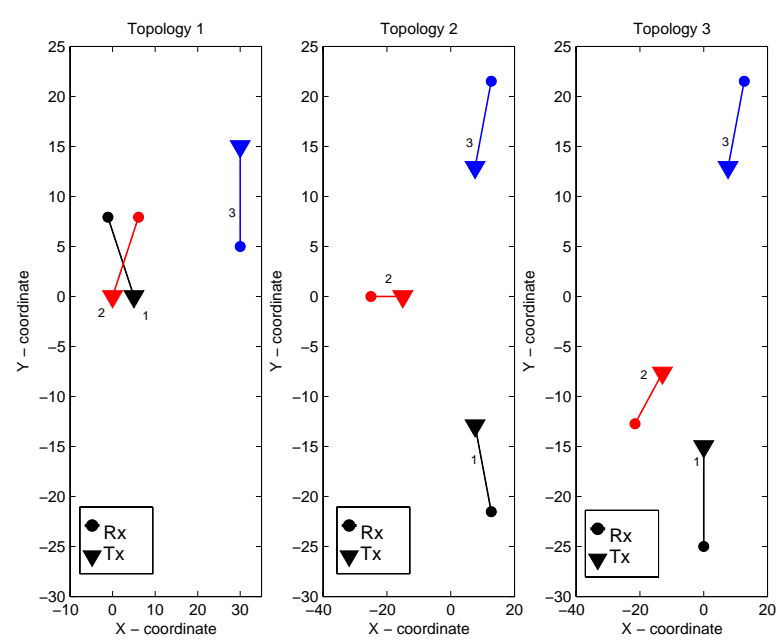

Fig. 1. Geometric plot of the three topologies

TABLE I

RAte Allocation For the THREE STRATEgies FOR TOPOLOGY 1

\begin{tabular}{|l|l|l|l|l|}
\hline Coalition Structure & $R_{1}$ & $R_{2}$ & $R_{3}$ & Sum-rate \\
\hline Transferable Payoff Allocation Strategies (NBS and PF) \\
\hline$\{1,2,3\}_{N B S}$ & 1.4391 & 1.4346 & 1.0671 & 3.9408 \\
\hline$\{1,2,3\}_{P F}$ & 1.4372 & 1.4365 & 1.0671 & 3.9408 \\
\hline Non-Transferrable Payoff Strategy (ER) \\
\hline$\{1,2,3\}$ & 1.3136 & 1.3136 & 1.3136 & 3.9408 \\
\hline$\{1,2\},\{3\}$ & 1.4174 & 1.4174 & 0.9355 & 3.7703 \\
\hline$\{2,3\},\{1\}$ & 0.4170 & 0.2055 & 0.2055 & 0.8280 \\
\hline$\{3,1\},\{2\}$ & 0.2115 & 0.4129 & 0.2115 & 0.8359 \\
\hline$\{1\},\{2\},\{3\}$ & 0.4170 & 0.4129 & 0.9355 & 1.7654 \\
\hline Stable ER Coalition: $\{1,2\},\{3\}$ \\
\hline
\end{tabular}

in the core and whether there are any fair means of arriving at such an allocation. We address this question by treating rate allocation as a bargaining problem between $M$ users. The agreement set is the set of payoff profiles in the core while the disagreement set is the set of rates achievable by each user independently without cooperation, i.e., (for the channel and signalling considered) the interference channel rates

$$
R_{i}^{I C}=I\left(X_{i} ; Y_{i}\right)=\log _{2}\left\{1+\frac{P_{i}\left|h_{i, i}\right|^{2}}{1+\sum_{k \neq i} P_{k}\left|h_{k, i}\right|^{2}}\right\}
$$

In other words, when a player is in disagreement, it does not cooperate with any other user. In this framework, players attempt to maximize the product of the utilities derived from cooperation. We propose the Nash bargaining solution (NBS) [15] and proportional fairness (PF) [16] as solution criteria to the bargaining model. Specifically, the Nash solution arrives at a point in the feasible rate region that maximizes the product of the rate gains achieved by each link through receiver cooperation over the interference channel rate $R_{i}^{I C}$. The product is maximized over the subset of the agreement set consisting of points that are strictly better than the disagreement payoffs for each player, i.e., $R_{i}>R_{i}^{I C} \forall i$. The proportional fair allocation simply maximizes the product of rates achieved over the feasible rate region. Both solutions 
TABLE II

RATE ALLOCATION FOR THE THREE STRATEGIES FOR TOPOLOGY 2

\begin{tabular}{|l|c|c|c|c|}
\hline Coalition Structure & $R_{1}$ & $R_{2}$ & $R_{3}$ & Sum-rate \\
\hline Transferable Payoff Allocation Strategies (NBS and PF) \\
\hline$\{1,2,3\}_{N B S}$ & 0.9988 & 0.9988 & 0.9988 & 2.9964 \\
\hline$\{1,2,3\}_{P F}$ & 0.9988 & 0.9988 & 0.9988 & 2.9964 \\
\hline Non-Transferrable Payoff Strategy (ER) \\
\hline$\{1,2,3\}$ & 0.9988 & 0.9988 & 0.9988 & 2.9964 \\
\hline$\{1,2\},\{3\}$ & 0.9671 & 0.9671 & 0.9673 & 2.9015 \\
\hline$\{2,3\},\{1\}$ & 0.9673 & 0.9671 & 0.9671 & 2.9015 \\
\hline$\{3,1\},\{2\}$ & 0.9671 & 0.9673 & 0.9671 & 2.9015 \\
\hline$\{1\},\{2\},\{3\}$ & 0.9673 & 0.9673 & 0.9673 & 2.9019 \\
\hline Stable ER Coalition: $\{1,2,3\}$ \\
\hline
\end{tabular}

TABLE III

RATE Allocation For THE THREE STRATEgIES FOR TOPOLOGY 3

\begin{tabular}{|l|c|c|c|c|}
\hline Coalition Structure & $R_{1}$ & $R_{2}$ & $R_{3}$ & Sum-rate \\
\hline Transferable Payoff Allocation Strategies (NBS and PF) \\
\hline$\{1,2,3\}_{N B S}$ & 0.9868 & 0.9868 & 1.0246 & 2.9982 \\
\hline$\{1,2,3\}_{P F}$ & 0.9994 & 0.9994 & 0.9994 & 2.9982 \\
\hline Non-Transferrable Payoff Strategy (ER) \\
\hline$\{1,2,3\}$ & 0.9994 & 0.9994 & 0.9994 & 2.9982 \\
\hline$\{1,2\},\{3\}$ & 0.9774 & 0.9774 & 0.9758 & 2.9306 \\
\hline$\{2,3\},\{1\}$ & 0.9230 & 0.9209 & 0.9209 & 2.7648 \\
\hline$\{3,1\},\{2\}$ & 0.9210 & 0.9231 & 0.9210 & 2.7651 \\
\hline$\{1\},\{2\},\{3\}$ & 0.9230 & 0.9231 & 0.9758 & 2.8219 \\
\hline Stable ER Coalition: $\{1,2,3\}$ \\
\hline
\end{tabular}

result in allocations that lie in the core [9]. We illustrate the receiver cooperation interference channel game with three example topologies and compare a simple equal rate (ER) apportioning strategy with the NBS and PF solutions.

\section{Coalitional Games in Linear Multiuser DETECTORS}

The formation of coalitions for the Gaussian multi-access channel has been studied in [17] where the interfering transmitters bargain for rates by threatening to transmit worst case jamming noise. Here, we focus on the use of linear multiuser detectors (MUDs) for forming coalitions between users in a MAC. We assume that the transmitters do not cooperate while the receivers decide to form linear multiuser detector coalitions to maximize their payoffs. In this case, the payoff achieved by each user is measured by its achieved SINR. Note that the rate achieved by a user is a monotonic increasing function of the SINR and maximizing SINR is equivalent to maximizing the rate.

We consider the decorrelating receiver [18] and the MMSE receiver [19] in the context of the MAC. While it is of interest to consider these detectors in the context of receiver cooperation in the interference channel model, we consider only the MAC to allow for a simplified analysis.

We consider a MAC channel with $M$ users communicating with one base station (BS) in a BPSK modulated, synchronized CDMA system with no power control. We denote the set of all users by $\mathcal{S}=\{1, \ldots, M\}$. Users are assumed to have been assigned signature sequences such that the correlation between

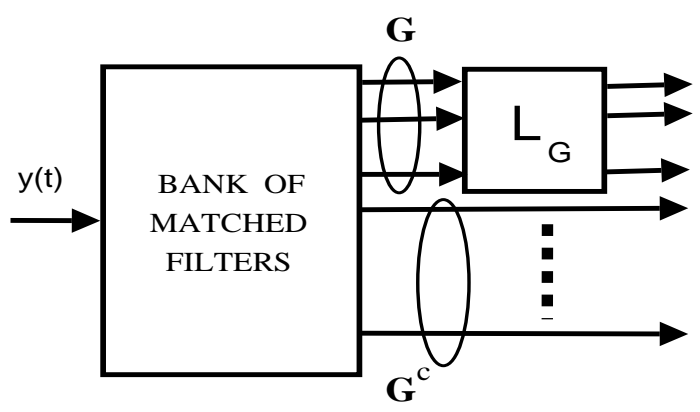

Fig. 2. A coalition $\mathcal{G}$ of users formed by a multiuser detector $\mathbf{L}_{\mathcal{G}}$

the sequences of any two users is $\rho$. The received signal at the BS is given by

$$
y(t)=\sum_{i=1}^{M} \sqrt{P} h_{i} b_{i} s_{i}(t)+\sigma n(t), \quad t \in[0, T]
$$

where $P$ is the common transmit power of each user, $h_{i}$ is channel gain from user $i$ to the BS and is assumed known at the BS, $b_{i} \in\{+1,-1\}$ is the bit transmitted by user $i$ in the bit interval $[0, T], s_{i}(t)$ is the signature sequence of user $i$ and $n(t)$ is an additive white Gaussian noise process with unit variance. The received signal in (7) is filtered through a bank of filters matched to the $M$ signature sequences resulting in the $M \times 1$ received signal vector $\mathbf{y} \in \mathbb{R}^{M}$ [6]

$$
\mathbf{y}=\mathbf{R A b}+\mathbf{n},
$$

where $\mathbf{R} \in \mathbb{R}^{M \times M}$ is the cross correlation matrix, $\mathbf{A}$ is a diagonal matrix of size $M \times M$ containing the received amplitudes of the $M$ users, $\mathbf{b}$ is an $M \times 1$ vector containing their transmitted bits and $\mathbf{n} \in \mathbb{R}^{M}$ is a Gaussian random vector with zero mean and covariance matrix $\sigma^{2} \mathbf{R}$. In a traditional linear MUD, the received vector $\mathbf{y}$ is put through a linear transformation $\mathbf{L}$ and the resulting vector $\mathbf{L y}$ is used for decoding the bits of the users. For the decorrelator, $\mathbf{L}=\mathbf{R}^{-1}$ and for the MMSE detector, $\mathbf{L}=\left(\mathbf{R}+\sigma^{2} \mathbf{A}^{-2}\right)^{-1}$. In the coalitional game formulated here, we assume that users form coalitions of multiuser detectors as shown in figure 2. Let $\mathcal{G} \subset \mathcal{S}$ be such a coalition. Then the received signal vector for this coalition can be written as

$$
\mathbf{y}_{\mathcal{G}}=\mathbf{R}_{\mathcal{G}} \mathbf{A}_{\mathcal{G}} \mathbf{b}_{\mathcal{G}}+\widetilde{\mathbf{R}}_{\mathcal{G}^{c}} \mathbf{A}_{\mathcal{G}^{c}} \mathbf{b}_{\mathcal{G}^{c}}+\mathbf{n}_{\mathcal{G}}
$$

where $\mathbf{R}_{\mathcal{G}} \in \mathbb{R}^{|\mathcal{G}| \times|\mathcal{G}|}$ is the cross correlation matrix of the users in $\mathcal{G}, \mathbf{A}_{\mathcal{G}} \in \mathbb{R}^{|\mathcal{G}| \times|\mathcal{G}|}$ is a diagonal matrix containing the received amplitudes of users in $\mathcal{G}, \mathbf{b}_{\mathcal{G}}$ is the vector of bits sent by users in $\mathcal{G}$ and $\mathbf{n}_{\mathcal{G}} \in \mathbb{R}^{|\mathcal{G}|}$ is a random Gaussian vector with zero mean and covariance matrix $\sigma^{2} \mathbf{R}_{\mathcal{G}}$. The matrix $\widetilde{\mathbf{R}}_{\mathcal{G}^{c}}$ is of dimension $|\mathcal{G}| \times\left|\mathcal{G}^{c}\right|$ and contains the cross correlations between the the signature sequences of users in $\mathcal{G}$ and those of users in $\mathcal{G}^{c}$, i.e., $\left(\widetilde{R}_{\mathcal{G}^{c}}\right)_{i j}=\rho, \forall i=1, \ldots,|\mathcal{G}|$ and $j=1, \ldots, M-|\mathcal{G}|, \mathbf{A}_{\mathcal{G}^{c}}$ is a $\left|\mathcal{G}^{c}\right| \times\left|\mathcal{G}^{c}\right|$ diagonal matrix containing the amplitudes of users not in $\mathcal{G}$ and $\mathbf{b}_{\mathcal{G}^{c}}$ 
is the $\left|\mathcal{G}^{c}\right| \times 1$ vector containing their transmitted bits. The linear MUD for the users in $\mathcal{G}$ puts the vector $\mathbf{y}_{\mathcal{G}}$ in (9) through the linear transformation $\mathbf{L}_{\mathcal{G}}$, where $\mathbf{L}_{\mathcal{G}}=\mathbf{R}_{\mathcal{G}}^{-1}$ for the decorrelating detector and $\mathbf{L}_{\mathcal{G}}=\left(\mathbf{R}_{\mathcal{G}}+\sigma^{2} \mathbf{A}_{\mathcal{G}}^{-} 2\right)^{-1}$ for the linear MMSE detector. Users within a coalition benefit from the interference suppression offered by their MUD. Since the SINR achieved by a user in a MUD cannot be shared with other users in the coalition, we model this scenario as a coalitional game with non-transferable payoff.

\section{Decorrelating Receiver}

We know that the decorrelating receiver will nullify all interference from users that are part of the same coalition, at the price of enhancing the noise and the interference from users outside the coalition. It can be shown that the SINR of a user in a decorrelating detector coalition $\mathcal{G}$ is given by [20]

$$
\begin{aligned}
x_{i}(\mathcal{G})= & \operatorname{SINR}_{i}^{\text {decorr }}(\mathcal{G}) \\
= & \frac{P_{i}}{\frac{\sigma^{2}}{1-\rho} \frac{1+\rho(|\mathcal{G}|-2)}{1+\rho(|\mathcal{G}|-1)}+\left[\frac{\rho}{1+\rho(|\mathcal{G}|-1)}\right]^{2} \sum_{j \in \mathcal{G}^{c}} P_{j}}, \\
& \forall i \in \mathcal{G},
\end{aligned}
$$

where $P_{k}=h_{k}^{2} P$ is the received power of user $k$ at the BS.

Theorem 8: In the decorrelating detector MAC game, the grand coalition is stable and sum-rate maximizing in the high SNR regime.

Proof: Recall that the core of a non-transferable payoff game is the set of all payoff profiles for which there is no coalition $\mathcal{G} \subset \mathcal{S}$ that can achieve a payoff vector $\underline{R}_{\mathcal{G}}=$ $\left(R_{i}\right)_{i \in \mathcal{G}}$ such that $R_{i}(\mathcal{G})>R_{i}(\mathcal{S}) \forall i \in \mathcal{G}$. If we can show that of all possible coalitions that a given user can be part of, it achieves the greatest payoff when it is in the grand coalition, and that this condition is true for all users in the MAC, then it follows that the grand coalition will be stable. The payoff of an arbitrary user $i$ when it is in the grand coalition is

$$
x_{i}(\mathcal{S})=\frac{P_{i}}{\frac{\sigma^{2}}{1-\rho} \frac{1+\rho(M-2)}{1+\rho(M-1)}}
$$

and it can be seen by comparing (11) with (10) that

$$
\lim _{\sigma^{2} \rightarrow 0} x_{i}(\mathcal{G})<\lim _{\sigma^{2} \rightarrow 0} x_{i}(\mathcal{S})
$$

Hence, in the high SNR regime, all users achieve the highest payoff in the grand coalition and therefore have no incentive to defect (any subset of users $\mathcal{G}$ would provide a smaller payoff to each of its members).

Since, in the high SNR regime, each user achieves its greatest payoff in the grand coalition, it follows that the sum of the rates achieved is the greatest among all coalition structures. The grand coalition is therefore sum-rate maximizing in the high SNR regime.

In general, however, there is no guarantee that the grand coalition of users should form or that the stable coalition structure should be the one that maximizes sum-rate. We illustrate this in the decorrelating detector game using an
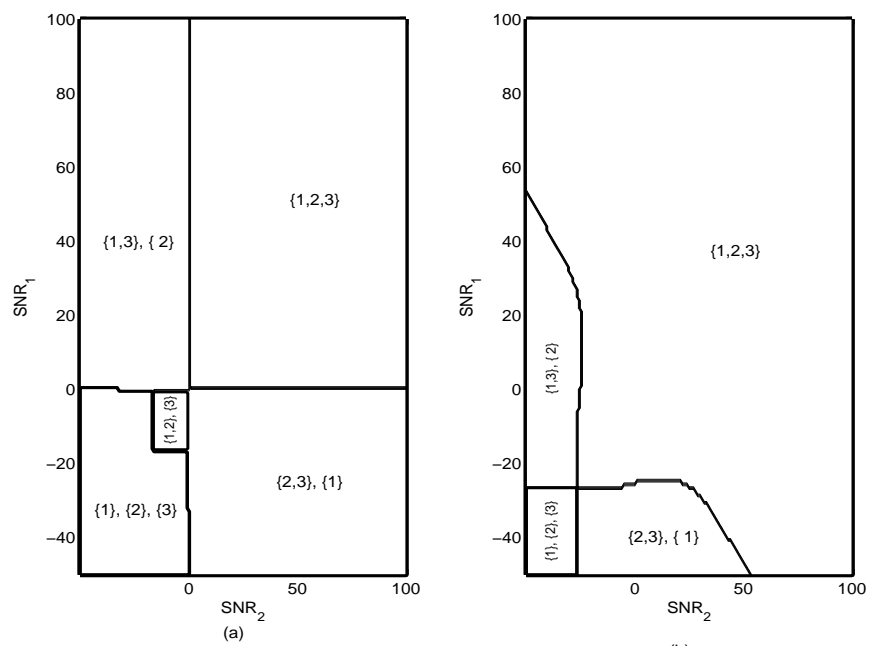

Fig. 3. (a) Stable coalition structures in a 3 user MAC (b) Sum-rate maximizing coalition structures $\left(\rho=0.001\right.$ and $\left.S R N_{c}=27 \mathrm{~dB}\right)$

example of a MAC with 3 users, keeping the channel of one of the users fixed and varying the channels of the other two users to show the stable coalition structure for various channel conditions. For the purpose of this illustration, we use a non-fading, path-loss channel model with path loss exponent $\alpha=3$. In figures 3(a) and 4(a), we show the stable coalition structures for different received SNRs of users 1 and 2, while the received SNR of user 3 is fixed at some value. The notation used is as follows: $\{1,2,3\}$ refers to the grand coalition, $\{1,3\},\{2\}$ refers to the coalition structure in which users 1 and 3 form a coalition while user 2 is alone and so on. An interesting question to ask here is whether the stable coalition structures formed also the ones that maximize the sum-rate. To answer this, we need to know the mapping of the SINR achieved by a user to its rate. We illustrate in figures $3(\mathrm{~b})$ and 4(b) with an example of such a mapping. Specifically, we map $S I N R_{i}(\mathcal{G})$, the SINR achieved by a user $i$ in a coalition $\mathcal{G}$ to its rate $R_{i}(\mathcal{G})$ as $R_{i}(\mathcal{G})=\log \left(1+\operatorname{SIN} R_{i}(\mathcal{G})\right)$. Under this mapping, the stable coalitions structures are not necessarily the ones that maximize the sum rate. It can be seen from the upper right hand corner of the plots that the grand coalition is both stable and sum-rate maximizing in the high SNR regime.

\section{Linear MMSE Receiver}

Unlike the decorrelating receiver, the linear MMSE receiver attacks both the noise and the interference and for a coalition $\mathcal{G}$, applies the linear transformation $\mathbf{L}_{\mathcal{G}}=\left[\mathbf{R}_{\mathcal{G}}+\sigma^{2} \mathbf{A}_{\mathcal{G}}^{2}\right]^{-1}$. It can be shown that the SINR $\gamma_{i}(\mathcal{G})$ of a user in a linear MMSE detector coalition $\mathcal{G}$ is given by [20]

$$
\gamma_{i}(\mathcal{G})=\frac{\left[\left(\mathbf{L}_{\mathcal{G}} \mathbf{R}_{\mathcal{G}}\right)_{i i}\right]^{2} P_{i}}{\left(\begin{array}{c}
\sigma^{2}\left(\mathbf{L}_{\mathcal{G}} \mathbf{R}_{\mathcal{G}} \mathbf{L}_{\mathcal{G}}\right)_{i i}+\rho^{2}\left[\left(\mathbf{L}_{\mathcal{G}} \mathbf{U}_{\mathcal{G}}\right)_{i}\right]^{2} \sum_{j \notin \mathcal{G}} P_{j} \\
+\sum_{j \in \mathcal{G}, j \neq i}\left[\left(\mathbf{L}_{\mathcal{G}} \mathbf{R}_{\mathcal{G}}\right)_{i j}\right]^{2} P_{j}
\end{array}\right)},
$$

where the second and third terms in the denominator of the above expression represent the effective interference to user $i$ from users outside the coalition $\mathcal{G}$ and that from other users 

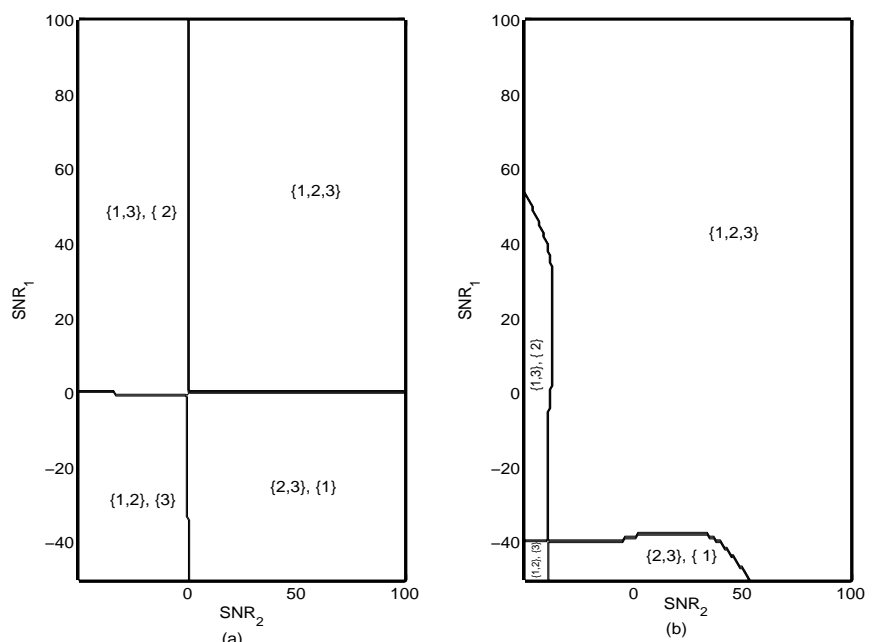

Fig. 4. (a) Stable coalition structures in a 3 user MAC (b) Sum-rate maximizing coalition structures $\left(\rho=0.001\right.$ and $\left.S R N_{c}=40 \mathrm{~dB}\right)$

in the MMSE coalition respectively. Here $\mathbf{U}_{\mathcal{G}}$ is the $|\mathcal{G}| \times 1$ vector $\mathbf{U}_{\mathcal{G}}=[1 \ldots 1]^{T}$.

Since minimizing the mean square error is equivalent to maximizing the SINR [19], it is straightforward to show that the grand coalition is always stable and is also sumrate maximizing, as long as the mapping of SINR to rate is monotonically increasing (non decreasing).

\section{CONCLusions And Future Work}

We have shown that cooperation between receivers in an interference channel and between single-user receivers in a MAC can lead to coalitions that are stable and can make optimal utilization of the shared spectrum. Our analysis is based on the premise that radios of the future will have the intelligence to make decisions that would involve such parameters as gains from cooperation and incentives to defect. As future work, we are interested in studying transmitter cooperation in wireless networks. We consider an interference channel such as the one in section III, where, in addition to receiver cooperation, transmitter cooperation is also possible. Given that the transmitters have available to them, an ideal, noise-free inter-user channel, we are interested in analyzing the coalitional game that arises from perfect transmitters cooperation. Transmitters are presented with the opportunity to form coalitions in which members jointly encode their transmit signals. Transmitters outside a coalition however act as interferers at the receivers. We assume that all receivers jointly decode their received signals.

It can be seen that such a game cannot be analyzed in characteristic function form. This is because the value of a coalition $\mathcal{G}$ will depend upon the actions of players outside it. In essence, any coalition $\mathcal{G}$ cannot make a decision about rejecting a proposed apportioning of value because it cannot determine with certainty, the payoff it would achieve if it broke away. Further analysis of the transmitter cooperation game in such a scenario remains an open problem.

It is possible, however, to convert the above game to characteristic function form by altering the definition of the value of a coalition to mean the maximum sum-rate it can achieve assuming it faces the worst possible interference from users outside it. This is analogous to the model proposed in [17] in the context of fair rate allocations to users in a MAC, where users who are dissatisfied with their rates can threaten to transmit worst case jamming noise to affect the rates of other users. Users in a breakaway coalition must therefore assume worst case interference from other users and determine their optimal joint transmission strategy. It remains to be seen whether the transmitter cooperation game can be guaranteed to have a non-empty core.

\section{ACKNOWLEDGEMENTS}

This work is supported in part by the NSF under grant number NeTS-0434854 and by the Defense Spectrum Office (DSO) of the Defense Information Systems Agency.

\section{REFERENCES}

[1] N. Bambos, "Toward power-sensitive network architectures in wireless communications: Concepts, issues, and design aspects," IEEE Pers. Commun., vol. 5, pp. 50-59, June 1998.

[2] I. Katzela and M. Naghshineh, "Channel assignment schemes for cellular mobile telecommunication systems: A comprehensive survey," IEEE Pers. Commun., vol. 3, no. 3, pp. 10-31, June 1996.

[3] L. Tassiulas and A. Ephremides, "Jointly optimal routing and scheduling in packet ratio networks," IEEE Trans. Inform. Theory, vol. 38, no. 1, pp. 165-168, Jan. 1992.

[4] A. Sendonaris, E. Erkip, and B. Aazhang, "User cooperation diversity part I: System description," IEEE Trans. Commun., vol. 51, no. 11, pp. 1927-1938, Nov. 2003.

[5] J. N. Laneman, D. N. C. Tse, and G. Wornell, "Cooperative diversity in wireless networks: efficient protocols and outage behavior,' IEEE Trans. Inform. Theory, vol. 50, no. 12, pp. 3062-3080, Dec. 2004.

[6] S. Verdu, Multiuser Detection. Cambridge University Press, 1998.

[7] O. Ileri, D. Samardzjia, T. Sizer, and N. B. Mandayam, "Demand responsive pricing and competitive spectrum allocation via a spectrum server," in IEEE Symp. New Frontiers in Dynamic Spectrum Access Networks, Baltimore, Maryland, Nov. 2005, pp. 194-202.

[8] C. Raman, R. D. Yates, and N. B. Mandayam, "Scheduling variable rate links via a spectrum server," in IEEE Symp. New Frontiers in Dynamic Spectrum Access Networks, Baltimore, Maryland, Nov. 2005, pp. 110 118.

[9] S. Mathur, L. Sankaranarayanan, and N. Mandayam, "Coalitional games in Gaussian interference channels," in (submitted to) Int. Symp. Inform. Theory, Seattle, Washington, July 2006, http://www.winlab.rutgers.edu/ $\sim$ suhas/msmisit2006.pdf.

[10] G. Owen, Game Theory. Academic Press, 1995.

[11] M. Osborne and A. Rubenstein, A Course in Game Theory. MIT Press, 1994.

[12] T. Sandholm, K. Larson, M. Anderson, O. Shehory, and F. Tohme, "Coalition structure generation with worst case guarantees," Artificial Intelligence, vol. 10, pp. 209-238, July 1999.

[13] A. B. Carleial, "Interference channels," IEEE Trans. Inform. Th., vol. 24, no. 1, pp. 60-70, Jan. 1978.

[14] E. Telatar, "Capacity of multi-antenna Gaussian channels," European Trans. Telecommunications, vol. 10, pp. 585-595, Nov. 1999.

[15] J. F. Nash, "The bargaining problem," Econometrica, vol. 18, no. 2, pp. 155-162, Apr. 1950.

[16] F. Kelly, "Charging and rate control for elastic traffic," Euro. Trans. Telecommun, vol. 8, pp. 33-37, Jan. 1997. 
[17] R. La and V. Anantharam, "A game-theoretic look at the Gaussian multiaccess channel," Advances in Network Information Theory, Proceedings of the March 2003 DIMACS workshop on Network Information Theory, DIMACS series in Discrete Mathematics and Theoretical Computer Science, vol. 66, pp. 37-42, Nov. 2003.

[18] R. Lupas and S. Verdu, "Linear multiuser detectors for synchronous code division multiple access channels," IEEE Trans. Inform. Theory, vol. 41, no. 4, pp. 1083-1096, Jan. 1989.

[19] U. Madhow and M. Honig, "MMSE interference suppression for direct sequence spread spectrum CDMA," IEEE Transactions on Communications, vol. 42, no. 12, pp. 3178-3188, Dec. 1994.

[20] Y. Li and A. Ephremides, "Linear multiuser detectors for incompletely known symmetric signals in CDMA systems," IEEE Trans. Inform. Theory, vol. 50, no. 6, pp. 1026-1040, June 2004. 